\title{
Enhancement of silicon oxidation rate due to tensile mechanical stress
}

\author{
Jui-Yuan Yen and Jenn-Gwo Hwu ${ }^{\text {a) }}$ \\ Department of Electrical Engineering, Rm 446, National Taiwan University, Taipei, Taiwan, \\ Republic of China
}

(Received 28 October 1999; accepted for publication 7 February 2000)

\begin{abstract}
Oxidation of silicon wafers under external mechanical stress was studied in this work. From the oxide thickness profile measured by an automatic ellipsometer, it was found that the oxidation kinetics of silicon was affected by the mechanical stress. The tensile stress strongly enhances the oxidation rate of silicon. A concept was proposed to explain this phenomenon by using a well-known physical $\mathrm{Si}-\mathrm{SiO}_{2}$ lattice model. The tensile stress in the silicon will enlarge the atom spacing of silicon and make the oxidation to be easier and faster. A simulated deformation of silicon substrate under tensile stress was also given to support this concept. This work is a direct evidence of the effect of mechanical stress on silicon oxidation. (C) 2000 American Institute of Physics. [S0003-6951(00)02714-5]
\end{abstract}

Stress-related problems have become a key issue of reliability in ultralarge-scale integration technology. ${ }^{1}$ Intrinsic stress during thermal oxidation is due to the mismatch of molecular volume and thermal expansion coefficients between $\mathrm{Si}$ and $\mathrm{SiO}_{2} \cdot{ }^{2-5}$ This kind of stress is compressive in the oxide and tensile in the silicon. ${ }^{6}$ Mechanical stress generally comes from the deposition of different kinds of films such as $\mathrm{SiO}_{2}$, poly-Si, $\mathrm{Si}_{3} \mathrm{~N}_{4}$, and metal electrodes. Structures of devices at sharp corner and interconnect edge exhibit high stress as well. Mechanical stress causes not only the dislocations of silicon substrate, cracking or failures of thin films, but also the degradation of electrical characteristics. Many studies have concentrated on the nonplaner silicon oxidation and stress-dependent oxidation kinetic theory by extending the well-known Deal-Grove model. ${ }^{7-9}$ It had been proposed that the compressive and tensile stresses from temperature nonuniformity or mechanical-applied forces could affect the oxidation kinetics and rates. ${ }^{10-13}$ However, there is still a lack of experimental evidence to prove this concept. In this work, we studied the direct effect of external mechanical stress on silicon oxidation.

Boron-doped (100), 3 in. silicon wafers with a resistivity of $1-10 \Omega \mathrm{cm}$ were used in this experiment. After standard RCA cleaning, the wafers were furnace oxidized in pure dry $\mathrm{O}_{2}$ for $10 \mathrm{~min}$ at $950^{\circ} \mathrm{C}, 1 \mathrm{~atm}$. Conventional furnace thermal oxidation was used here in order to prevent the factor of temperature nonuniformity. Figure 1 shows the schematic diagram of the experimental setup. The wafers were placed vertically on a quartz boat. A small slice of quartz of about $20 \mathrm{~g}$ was put across the middle of two adjacent wafers such that the two adjacent wafers sustain compressive and tensile stresses separately. The external applied force was examined not to cause visible dislocations or defects on silicon wafers. After oxidation, the oxide thickness was measured by an automatic ellipsometer with an accuracy of $\pm 5 \AA$. The data were measured in $3 \mathrm{~mm}$ pitch so that one can see the entire oxide thickness profile of a silicon wafer.

Figures 2(a) and 2(b) show the oxide thickness profiles

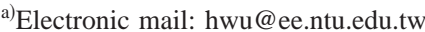

of the silicon wafer under compressive and tensile stress, respectively. As can be seen from this figure, the wafer with tensile stress has a significant increase of oxide thickness around the portion that stress applied. That is to say, a tensile stress applied on the silicon substrate enhances the oxidation rate significantly, while a compressive stress does not have a similar effect. It is believed that this phenomenon was mainly caused by the external applied stress since the temperature in the conventional furnace was assumed to be constant. It can be inferred that the mechanical stress affects the oxidation kinetics. In order to investigate the quality of silicon wafers under external mechanical stress, we used preferential silicon etching solution of Yang's ${ }^{14-17}$ to etch wafer after oxide removal. By comparing the microscope pictures $(100 \times)$ of etch pits, we saw no difference of etching characteristics between the experimental and reference wafers. Only the places near the edge where the wafer contacted with quartz boat showed slip dislocations. Therefore, it is believed that the slip dislocations are not severe in this work and is also not responsible for the enhancement of oxidation rate.

According to the well-known physical model of $\mathrm{Si}-\mathrm{SiO}_{2}$ interface, dangling bond occurred when the silicon lattice was not terminated with oxygen atom. When mechanical stress was applied during silicon oxidation, the spacing between silicon atoms was changed. It is suggested that tensile stress in the silicon will enlarge the atom spacing of silicon

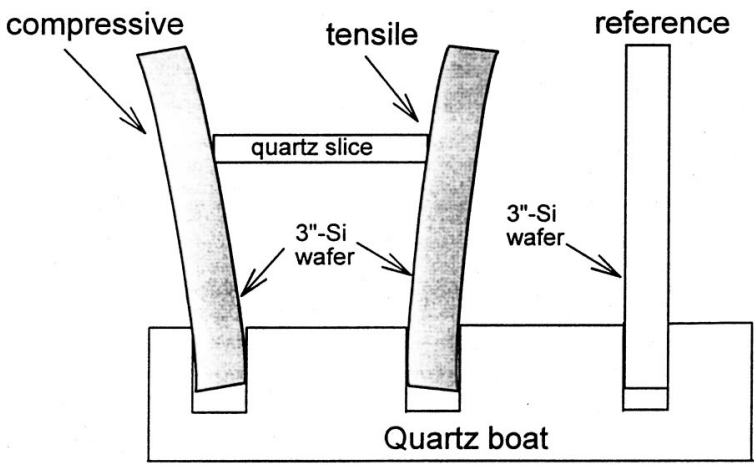

FIG. 1. Experimental setup of furnace oxidation with tensile and compressive stresses applied on wafers. 


\section{Compressive Stress}

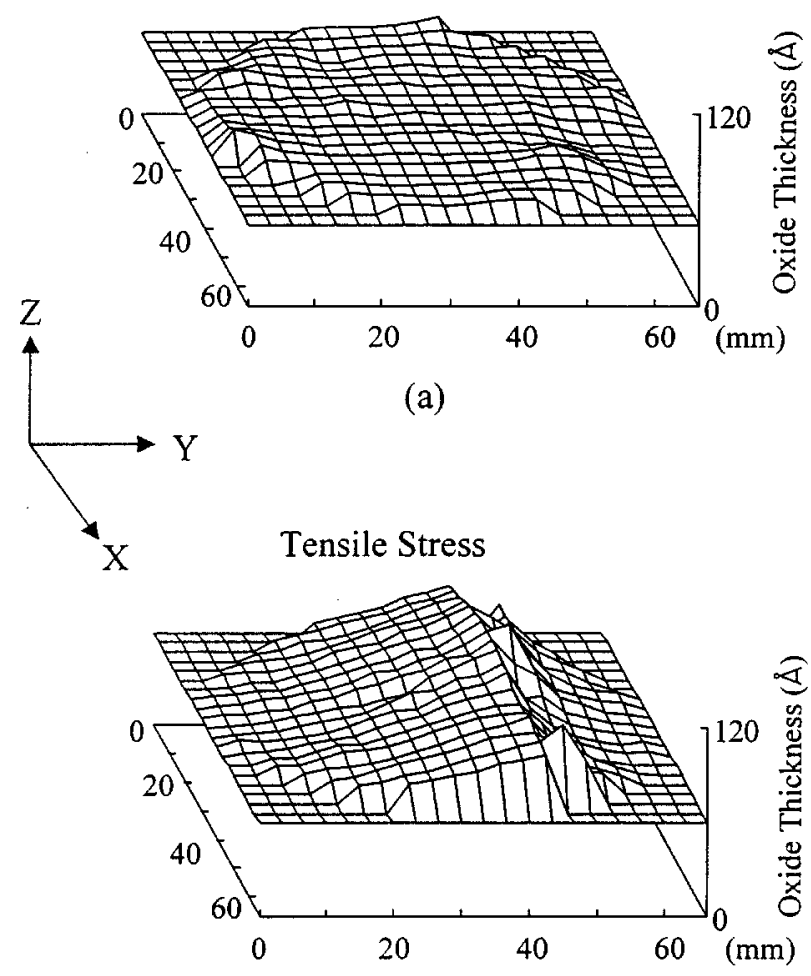

(b)

FIG. 2. Oxide thickness distributions of wafers under (a) compressive stress and (b) tensile stress.

and make the oxidation to be easier and faster. But a compressive stress will shorten the atom spacing of silicon and make the oxidation to be harder and slower. In this work, the experimental result of tensile stress supports this concept while that of compressive stress does not have a significant effect. The latter is mainly due to reason that compressive stress was applied on the backside of silicon substrate.

From Fig. 2(b), the oxide thickness distribution of silicon wafer under tensile stress has an increasing feature across the wafer. It is suggested that this phenomenon be related to the deformation of silicon wafer. Figure 3 shows the simulated deformation distribution of silicon wafer under tensile stress in the $X$ and $Y$ directions, i.e., $U_{x}$ and $U_{y}$, respectively, by using the ANSYS $^{\odot} 5.5$ simulation software. The mechanical stress applied in the $Z$ direction causes deformations in the $X$ and $Y$ directions as well. The deformations in the $X$ and $Y$ directions represent the variations of spacing between silicon atoms. Since the spacing of silicon atoms is a main factor in determining oxidation kinetics and rate, the simulation provides a meaningful concept to explain the experimental results. It is believed that the mechanical force applied in the $Z$ direction produces a more serious deformation of silicon wafer in the $Y$ direction than in the $X$ direction. The former dominates the oxidation kinetics in this work and has a significant effect on oxide growth rate.

Besides earlier discussions, there were literatures which addressed microscopic mechanisms, i.e., interstitial and vacancy diffusion mechanisms in silicon under stress. ${ }^{18-20}$ These studies provided useful explanation for our experimental results. According to Antonelli and Bernholc's
Simulation (Tensile Stress)

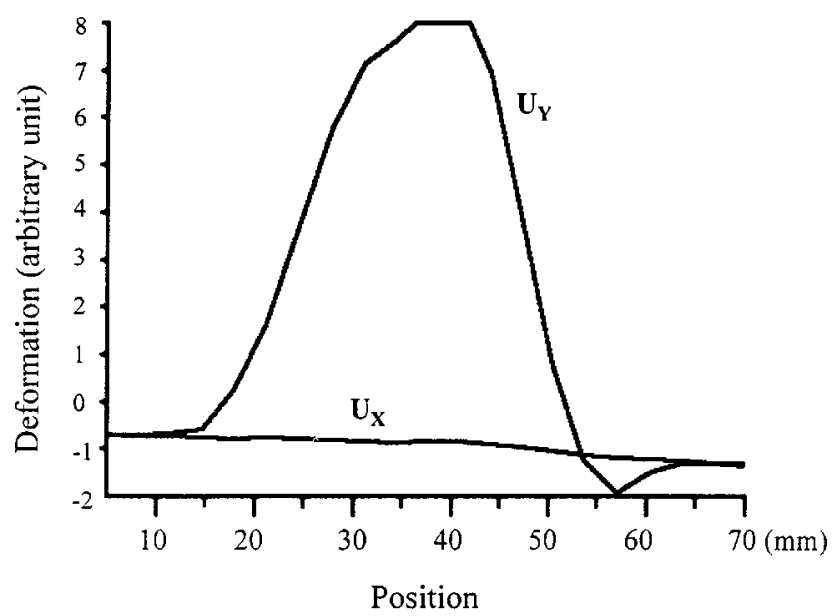

FIG. 3. Simulated deformation distributions of silicon wafer under tensile stress in the $X$ and $Y$ directions.

model,${ }^{18}$ the tensile stress could possibly expand the volume of silicon crystal and increase the vacancy density. When a vacancy is created, a $\mathrm{Si}$ atom leaves a lattice site and migrates to the surface. Therefore, the oxidation becomes easier and the oxidation rate increases.

In conclusion, silicon oxidation with external mechanical stress applied is useful in studying the mechanical stressinduced oxidation rate. With the aid of deformation analysis by simulation, one can know that an enlarged atom spacing of silicon will enhance the oxidation rate. There is another meaningful concept from the literature to realize the experiment results by using the microscopic vacancy diffusion mechanism. Our work shows that the oxide thickness is not only a function of temperature, but also of stress. This is important to investigate the oxide quality due to stress especially for ultra thin thickness regime.

This work was supported by the National Science Council of the Republic of China under Contract No. NSC 892218-E-002-016.

${ }^{1}$ S. M. Hu, J. Appl. Phys. 70, R53 (1991).

${ }^{2}$ R. J. Jaccodine and W. A. Schlegel, J. Appl. Phys. 37, 2429 (1966).

${ }^{3}$ E. P. EerNisse, Appl. Phys. Lett. 30, 290 (1977).

${ }^{4}$ E. P. EerNisse, Appl. Phys. Lett. 35, 8 (1979).

${ }^{5}$ T. Y. Tan and U. Gosele, Appl. Phys. Lett. 39, 86 (1981).

${ }^{6}$ E. P. Jacobs and G. Dorda, Surf. Sci. 73, 357 (1978).

${ }^{7}$ H. Matsumoto and M. Fukuma, IEEE Trans. Electron Devices ED-32, 132 (1985).

${ }^{8}$ D. B. Kao, J. P. McVittie, W. D. Nix, and K. C. Saraswat, IEEE Trans. Electron Devices ED-34, 1008 (1987).

${ }^{9}$ D. B. Kao, J. P. McVittie, W. D. Nix, and K. C. Saraswat, IEEE Trans. Electron Devices ED-35, 25 (1988).

${ }^{10}$ R. Deaton and H. Z. Massoud, J. Appl. Phys. 70, 3588 (1991).

${ }^{11}$ A. Minhalyi, R. J. Jaccodine, and T. J. Delph, Appl. Phys. Lett. 74, 1981 (1999).

${ }^{12}$ B. E. Deal and A. S. Grove, J. Appl. Phys. 36, 3770 (1965).

${ }^{13}$ T. J. Delph, J. Appl. Phys. 83, 786 (1998).

${ }^{14}$ F. S. d'Aragona, J. Electrochem. Soc. 119, 948 (1972).

${ }^{15}$ M. W. Jenkins, J. Electrochem. Soc. 124, 757 (1977).

${ }^{16}$ D. G. Schimmel, J. Electrochem. Soc. 126, 479 (1979).

${ }^{17}$ K. H. Yang, J. Electrochem. Soc. 131, 1140 (1984).

${ }^{18}$ A. Antonelli and J. Bernholc, Phys. Rev. Lett. 44, 593 (1980).

${ }^{19}$ N. E. B. Cowern, J. Appl. Phys. 64, 4484 (1988).

${ }^{20}$ A. Antonelli, E. Kaxiras, and D. J. Chadi, Phys. Rev. Lett. 81, 2088 (1998). 